Computational Mechanics manuscript No.

(will be inserted by the editor)

\section{A coupling strategy for adaptive local refinement in space and time with a fixed global model in explicit dynamics}

\author{
Omar Bettinotti · Olivier \\ Allix · Benoît Malherbe
}

Received: date / Accepted: date

\begin{abstract}
In dynamics, Domain Decomposition Methods (DDMs) enable one to use different spatial and temporal discretizations depending on the physical phenomenon being taken into account. Thus, DDMs provide the analyst with key tools for dealing with problems in which phenomena occur on different temporal and spatial scales. This paper focuses on a less intrusive variation of this type of method which enables the global (industrial) mesh to remain unchanged while the local problem is being refined in space and in time where needed. This property is particularly useful in the case of a local problem whose localization evolves rapidly with time, as is the case for delamination. The downside is that the technique is iterative. The method is presented in the context of linear explicit dynamics, but, as with domain decomposition, its extension to other integration schemes and to nonlinear problems should be possible.
\end{abstract}

Keywords global-local coupling - substitution method · explicit dynamics - multi-time-step analysis $\cdot$ nonintrusive technique

\section{Introduction}

Current design tools enable one to determine whether a given structure can sustain a specified impact load within some appropriate safety margins. A detailed study of the response

O. Bettinotti, O. Allix

LMT-Cachan

Tel.: +33-1-47407794

Fax: +33-1-47402785

E-mail: $\{$ bettinotti, allix $\} @ 1 m t . e n s-c a c h a n . f r$

B. Malherbe

Airbus Operations SAS

Tel.: +33-5-61936326

E-mail: benoit.malherbe@ airbus.com up to failure often requires taking into account phenomena which spread over multiple spatial and temporal scales. For example, this is the case when one wishes to predict the extensive delaminations which may occur in multilayered composites under impact. One of the objectives of Airbus is to address this problem through the nonintrusive implementation of a damage mesomodel for laminates [1] [2]. In the case of large structures, this would require optimal finite element discretizations using state-of-the-art localized adaptive mesh refinement. Often, unfortunately, the implementation of hp-adaptivity into commercial finite element analysis software is extremely difficult or even, in many cases, impossible. Also, the treatment of localized impact loads requires special attention and, in general, cannot be carried out automatically in conventional FEA software. Moreover, if such loads are applied to meshes which are designed to capture only the global response of the structure, the error in the finite element solution may be significant, even far from the localized features, due to what is known as the pollution error [3].

In order to overcome these limitations, first, monolithic strategies were developed (e.g. implicit-explicit algorithms [5] [25] or multi-time-step methods [33] [14]). More recently, this type of approach has led to the development of non-overlapping dual domain decomposition methods [20] [10] [7] or overlapping methods like in the Arlequin framework [19]. These methods enable one to use different meshes, time steps and integration schemes in different parts of the structure being studied. They are quite suitable for problems in which the zones to be refined are fixed. However, in the case of the problems we are considering, these zones may evolve rapidly, which, in a domain-decomposition-like approach, would require one to remesh the whole structure. This would be both difficult and expensive and would require rather intrusive developments.

Let us note that, today, there is growing interest in nonintrusive methods which enable one to deal with multiscale problems using an industrial code as the global solver. Domain decomposition methods are often used as bases of globallocal approaches [35], such as the one presented in [21], and lend themselves to the use of accelerated schemes in the case of nonlinear problems [12]. An interesting approach to the development of a nonintrusive version of the domain decomposition method consists in the replacement of the local model where needed. This is what we did in statics in [18], and improved in [17]. Subsequently, this technique was applied successfully to the coupling of a mesomodel and a micromodel of a laminate in [13]. The same approach was extended to stochastic problems [8] using the Proper Generalized Decomposition (PGD) method [26] [32]. In [30], in the context of thermal shock problems, another approach than domain decomposition was used: a nonintrusive version of the Generalized Finite Element Method (GFEM) was de- 
veloped using the properties of the partition of unity. A development of this approach for 3D fracture mechanics was presented in [22]. The main characteristic of this approach is the development of nonintrusive enrichment techniques, leading to the use of local-global shape functions.

This paper focuses on the development of the substitution method in the linear explicit dynamic case. The basic idea consists in establishing an iterative coupling between a global analysis applied to the entire structure and an overlapping local analysis applied to the zones of interest. The local analysis is activated only when necessary by imposing the continuity of the velocities at some interfaces of the local domain and performing an iterative correction in order to achieve equilibrium. The objective of the method is to leave the domain topology of the entire structure unchanged and to apply the remeshing strategy only to the local analysis of the zones of interest. The downside is that this method is iterative.

The paper is organized as follows: after this introduction, the finite element formulation of the reference problem and its temporal integration scheme are described in Section 2. A first substitution approach enabling the global mesh and the associated time step to remain unchanged is presented in Section 3 and compared to the domain decomposition method proposed in [28] based on academic 2D examples. As in [10], we find that, regardless of the tolerance criteria used for the coupling between the global and local meshes, some energy imbalance remains. Then, in Section 4, we develop a second substitution approach, whose implementation follows [31] and [28], which has the advantage that its energy conservation properties, which are linked directly to the tolerance criteria being used for the coupling, can be controlled. As a consequence of the stability study, both the two versions of the substitution method have been modified in order to solve the instability issues: these modifications are described in Section 5. At the end, in Section 6, we present some conclusions and discuss the perspectives of the method.

\section{The reference problem}

The initial boundary value problem of domain $\Omega$ shown in Figure 1 is:

$$
\begin{array}{ll}
\rho \ddot{\mathbf{u}}=\operatorname{div}(\sigma) & \text { over } \Omega \times\left[t_{0}, t_{\text {end }}\right] \\
\mathbf{u}=\overline{\mathbf{u}} & \text { along } \partial \Omega_{u} \times\left[t_{0}, t_{\text {end }}\right] \\
\sigma \cdot \mathbf{n}=\mathbf{f}_{\text {surf }} & \text { along } \partial \Omega_{f} \times\left[t_{0}, t_{\text {end }}\right] \\
\{\mathbf{u}, \dot{\mathbf{u}}\}=\left\{\mathbf{u}_{0}, \mathbf{v}_{0}\right\} & \text { over }\left.\Omega\right|_{t_{0}}
\end{array}
$$

where $\rho$ is the density of the material, $\sigma$ the stress tensor, $\mathbf{f}_{\text {surf }}$ the surface load and $\mathbf{n}$ the unit normal to boundary $\partial \Omega_{f}$. The initial and final times are denoted $t_{0}$ and $t_{\text {end }}$. The stress tensor $\sigma$ is related to the strain tensor $\varepsilon$ through a material constitutive law under plane strain assumption. The body forces are ignored.

The first line of System (1) represents the momentum balance as a function of the displacements $\mathbf{u}$; the second and third lines represent respectively the essential and natural boundary conditions, and the fourth line represents the initial conditions.

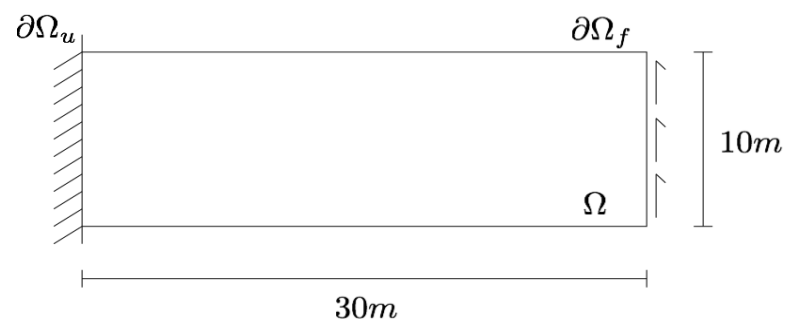

Fig. 1: The domain of definition of the plane strain problem

The boundaries $\partial \Omega_{u}$, along which the displacements are prescribed, and $\partial \Omega_{f}$, along which surface loads are applied, do not overlap; their union constitutes the entire boundary $\partial \Omega$ of the problem. Thus, $\partial \Omega_{f} \cup \partial \Omega_{u}=\partial \Omega$ and $\partial \Omega_{f} \cap$ $\partial \Omega_{u}=\varnothing$.

\subsection{Finite element formulation: space and time} discretization

Problem (1) can be expressed, in weak formulation, as:

$$
\begin{aligned}
& \int_{t_{0}}^{t_{\text {end }}} \int_{\Omega} \rho \ddot{\mathbf{u}} \delta \mathbf{u} d \Omega d t+\int_{t_{0}}^{t_{\text {end }}} \int_{\Omega} \sigma(\mathbf{u}): \varepsilon(\delta \mathbf{u}) d \Omega d t= \\
= & \int_{t_{0}}^{t_{\text {end }}} \int_{\partial \Omega_{f}} \mathbf{f}_{\text {surf }} \delta \mathbf{u} d \Gamma d t \quad \forall \delta \mathbf{u}=\mathbf{0} \text { along } \partial \Omega_{u} \times\left[t_{0}, t_{\text {end }}\right]
\end{aligned}
$$

Using a displacement-based finite element method [24] [4] [36] with the heterogeneous spatial discretization described in Figure 2, Equation (2) becomes:

$$
\begin{array}{ll}
\mathbf{M} \ddot{\mathbf{U}}=\mathbf{F}_{\text {ext }}-\mathbf{F}_{\text {int }} & \text { over } \Omega_{1}^{h} \cup \Omega_{2}^{h} \times\left[t_{0}, t_{\text {end }}\right] \\
\mathbf{U}=\overline{\mathbf{U}} & \text { along } \partial \Omega_{u} \times\left[t_{0}, t_{\text {end }}\right] \\
\{\mathbf{U}, \dot{\mathbf{U}}\}=\left\{\mathbf{U}_{0}, \mathbf{V}_{0}\right\} & \text { over }\left.\Omega_{1}^{h} \cup \Omega_{2}^{h}\right|_{t_{0}}
\end{array}
$$

where $\mathbf{M}$ is the lumped mass matrix, $\mathbf{F}_{\text {ext }}$ and $\mathbf{F}_{\text {int }}$ are respectively the external and internal force vectors, and $\Omega_{1}^{h} \cup \Omega_{2}^{h}$ represents the union of two different homogeneous finite element discretizations which, together, span the whole domain $\Omega$.

The more refined mesh $\Omega_{1}^{h}$ is defined near boundary condition $\partial \Omega_{u}$ in order to limit the locking effect.

An explicit time integration scheme using the central difference method is applied. For a given spatial discretization, 


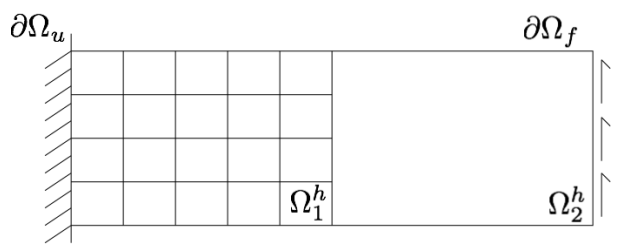

(a) spatial mesh

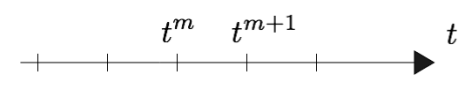

(b) temporal discretization

Fig. 2: The heterogeneous discretization

the nodal displacements, velocities and accelerations at time $t^{m}$ are defined as:

$\mathbf{U}^{m}=\mathbf{U}\left(t^{m}\right) ; \quad \mathbf{V}^{m}=\dot{\mathbf{U}}\left(t^{m}\right) ; \quad \mathbf{A}^{m}=\ddot{\mathbf{U}}\left(t^{m}\right)$.

This time integration scheme is not unconditionally stable, so the choice of the time step size is governed by the CFL condition [11]. In this paper, even if in impact and blast problems the mesh distortion leads to modify the time step during the analysis [23], the simple situation in which the global time step is fixed throughout all the computation is considered. Thus, one estimates a critical time step $\Delta t_{\mathrm{cr}}$ (see, for example, [16]) and one applies a fixed time step $\Delta t=t^{m+1}-t^{m}$ throughout the analysis:

$\Delta t \leq 0.8 \Delta t_{\mathrm{cr}} \quad$ with $\quad \Delta t_{\mathrm{cr}}=\frac{2}{\omega_{\max }}$

where $\omega_{\max }$ is the maximum eigenfrequency of the problem, that is discretized in space by the finite elements $e$ :

$\omega_{\max }=\max _{e}\left\{\omega_{e}\right\}$

such that the time step $\Delta t$ is governed by the refined part $\Omega_{1}^{h}$ of the mesh.

Using the central difference scheme written in the Newmark formulation [29] (with parameters $\beta=0$ and $\gamma=1 / 2$ ), the calculation of the solution at each time $t^{m+1}$ involves three sequential steps:

1. the updating of the displacements $\mathbf{U}^{m+1}$ and the definition of the predictive velocities ${ }^{p} \mathbf{V}^{m}$ :

$$
\begin{array}{ll}
\mathbf{U}^{m+1}=\mathbf{U}^{m}+\Delta t \mathbf{V}^{m}+\frac{\Delta t^{2}}{2} \mathbf{A}^{m} & \text { over } \Omega_{1}^{h} \cup \Omega_{2}^{h} \\
{ }^{p} \mathbf{V}^{m}=\mathbf{V}^{m}+\frac{\Delta t}{2} \mathbf{A}^{m} & \text { over } \Omega_{1}^{h} \cup \Omega_{2}^{h}
\end{array}
$$

2. the calculation of the accelerations $\mathbf{A}^{m+1}$ through the resolution of the momentum balance equation and the application of the essential boundary conditions:

$$
\begin{array}{ll}
\mathbf{M A}^{m+1}=\mathbf{F}_{\text {ext }}^{m+1}-\mathbf{F}_{\text {int }}\left(\mathbf{U}^{m+1}\right) & \text { over } \Omega_{1}^{h} \cup \Omega_{2}^{h} \\
\mathbf{U}^{m+1}=\overline{\mathbf{U}}^{m+1} & \text { along } \partial \Omega_{u}
\end{array}
$$

3. the updating of the velocities $\mathbf{V}^{m+1}$ :

$$
\mathbf{V}^{m+1}={ }^{p} \mathbf{V}^{m}+\frac{\Delta t}{2} \mathbf{A}^{m+1} \text { over } \Omega_{1}^{h} \cup \Omega_{2}^{h}
$$

It is to be noted that the central difference scheme written in the Newmark formulation is equivalent to the Verlet integration scheme [34] taking ${ }^{p} \mathbf{V}^{m} \equiv \mathbf{V}^{m+\frac{1}{2}}$.

With this monolithic approach, the time step imposed by the refined mesh $\Omega_{1}^{h}$ is also used in the coarse mesh $\Omega_{2}^{h}$ where such a small time step would not be necessary. This leads to a significant loss of efficiency, especially if the extent of the coarse domain $\Omega_{2}^{h}$ is large.

\subsection{Finite element formulation with domain decomposition}

The domain decomposition approach enables one to use different time steps for different meshes, for example with the discretization shown in Figure 3.

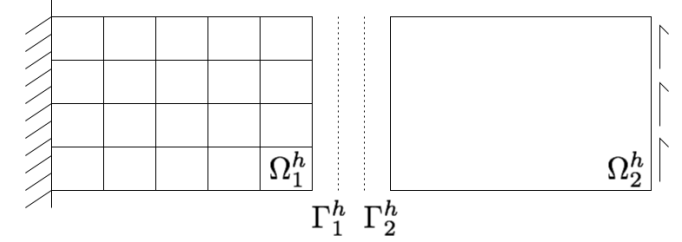

(a) spatial meshes

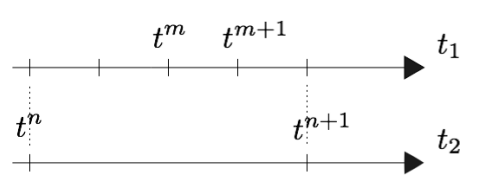

(b) temporal discretizations

Fig. 3: Discretization with domain decomposition

The heterogeneous discretization of Figure 2 is divided into two subdomains: $\Omega_{1}^{h}$ with a refined homogeneous mesh, and $\Omega_{2}^{h}$ with a coarse homogeneous mesh. The kinematic continuity between the two subdomains is enforced through Lagrange multipliers $\Lambda$ and the compatibility between the meshes is handled through linear spatial interpolation.

A refined time step $\Delta t_{1}=t^{m+1}-t^{m}$ is applied only to the subdomain with the fine mesh $\left(\Omega_{1}^{h}\right)$, while a coarse time step $\Delta t_{2}=t^{n+1}-t^{n}$ is applied to the subdomain with the coarse mesh $\left(\Omega_{2}^{h}\right)$. The ratio of the two time steps is chosen to be the ratio of the two mesh sizes, so that $\Delta t_{2}=M \Delta t_{1}$ and $t_{\text {end }}-t_{0}=N \Delta t_{2}$.

The dual algorithm introduced in [10] is applied to the domain decomposition of Figure 3 by setting the initial conditions $\left\{\mathbf{U}_{1}^{0}, \mathbf{V}_{1}^{0}\right\}=\left\{\mathbf{U}_{0}, \mathbf{V}_{0}\right\}$ over $\Omega_{1}^{h}$ and $\left\{\mathbf{U}_{2}^{0}, \mathbf{V}_{2}^{0}\right\}=\left\{\mathbf{U}_{0}, \mathbf{V}_{0}\right\}$ over $\Omega_{l}^{h}$ and using the following equations: 
1. $\forall m \in\{1, \ldots, M \cdot N\}:$

$$
\begin{array}{ll}
\mathbf{M}_{1} \mathbf{A}_{1}^{m+1}=\mathbf{C}_{1}^{T} \Lambda^{m+1}-\mathbf{F}_{1, \text { int }}^{m+1} & \text { over } \Omega_{1}^{h} \\
\mathbf{U}_{1}^{m+1}=\overline{\mathbf{U}}^{m+1} & \text { along } \partial \Omega_{u}
\end{array}
$$

where $\mathbf{C}_{1}$ is the Boolean connectivity matrix which extracts the nodal values from subdomain $\Omega_{1}^{h}$ to interface $\Gamma_{1}^{h}$ (see appendix A);

2. $\forall n \in\{1, \ldots, N\}$ :

$$
\mathbf{M}_{2} \mathbf{A}_{2}^{n+1}=\mathbf{F}_{\mathrm{ext}}^{n+1}-\mathbf{C}_{2}^{T} \tilde{\Pi}_{h}^{1}\left(\Lambda^{n+1}\right)-\mathbf{F}_{2, \text { int }}^{n+1} \text { over } \Omega_{2}^{h}
$$

where $\tilde{\Pi}_{h}^{1}(\cdot)$ is a linear spatial projection operator from the nodes of interface $\Gamma_{1}^{h}$ to the nodes of interface $\Gamma_{2}^{h}$ and $\mathbf{C}_{2}$ is the Boolean connectivity matrix which extracts the nodal values from subdomain $\Omega_{2}^{h}$ to interface $\Gamma_{2}^{h}$ (see appendix A);

3. $\forall m \in\{1, \ldots, M \cdot N\}$ :

$$
\mathbf{C}_{1} \mathbf{V}_{1}^{m+1}=\Pi_{h}^{1}\left(\mathbf{C}_{2} \Pi_{t}^{1}\left(\mathbf{V}_{2}^{n, n+1}\right)\right) \text { along } \Gamma_{1}^{h}
$$

where $\Pi_{h}^{1}(\cdot)$ is the linear spatial interpolation operator from the nodes of interface $\Gamma_{2}^{h}$ to the nodes of interface $\Gamma_{1}^{h}$ (see appendix A) and $\Pi_{t}^{1}(\cdot)$ is the linear temporal interpolation operator from times $t^{n}, t^{n+1}$ to times $t^{m}$, $t^{m+1}$.

As shown in [31] and [27], the approximation of the velocities in the coarse subdomain by linear temporal interpolation leads to numerical dissipation. An energy preserving algorithm was presented in [31] and in [28]. This second algorithm is defined by the following equations:

1. $\forall m \in\{1, \ldots, M \cdot N\}:$

$$
\begin{array}{lr}
\mathbf{M}_{1} \mathbf{A}_{1}^{m+1}=\mathbf{C}_{1}^{T} \Pi_{t}^{1}\left(\Lambda^{n, n+1}\right)-\mathbf{F}_{1, \text { int }}^{m+1} & \text { over } \Omega_{1}^{h} \\
\mathbf{U}^{m+1}=\overline{\mathbf{U}}^{m+1} & \text { along } \partial \Omega_{u}
\end{array}
$$

2. $\forall n \in\{1, \ldots, N\}$ :

$$
\mathbf{M}_{2} \mathbf{A}_{2}^{n+1}=\mathbf{F}_{\mathrm{ext}}^{n+1}-\mathbf{C}_{2}^{T} \tilde{\Pi}_{h}^{1}\left(\Lambda^{n+1}\right)-\mathbf{F}_{2, \text { int }}^{n+1} \text { over } \Omega_{2}^{h}
$$

3. $\forall n \in\{1, \ldots, N\}$ :

$$
\mathbf{C}_{1} \mathbf{V}_{1}^{n+1}=\Pi_{h}^{1}\left(\mathbf{C}_{2} \mathbf{V}_{2}^{n+1}\right) \text { along } \Gamma_{1}^{h}
$$

\section{The substitution method}

Here, the problem described in Figure 2 is solved using the substitution method: a global analysis is carried out with a coarse discretization $\Omega_{g}^{h}$ over the whole domain (Figure 4) and a local analysis is carried out with a more refined discretization $\Omega_{l}^{h}$ near boundary $\partial \Omega_{u}$ (Figure 5). The continuity of the velocities between the two analyses is ensured along the local interface $\Gamma_{l}^{h}$ through the Lagrange multipliers $\Lambda$ discussed in Section 3.4 and an iterative scheme is applied in order to reach the equilibrium between the two analyses.

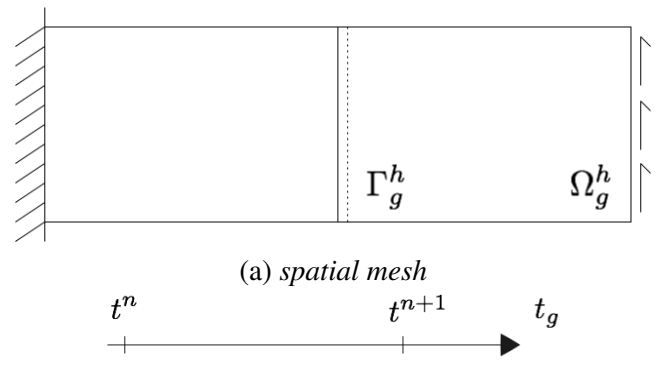

(b) temporal discretization

Fig. 4: Discretization of the whole domain (global analysis)

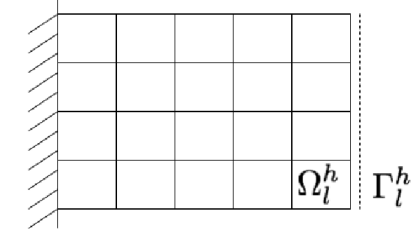

(a) spatial mesh

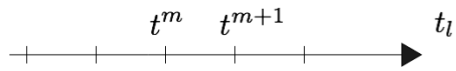

(b) temporal discretization

Fig. 5: Discretization of a part of the domain (local analysis)

Similar to the domain decomposition approach, the time steps are defined such that $\Delta t_{g}=M \Delta t_{l}$.

\subsection{Finite element formulation}

Applying the initial conditions $\left\{\mathbf{U}_{g}^{0}, \mathbf{V}_{g}^{0}\right\}=\left\{\mathbf{U}_{0}, \mathbf{V}_{0}\right\}$ over $\Omega_{g}^{h}$ and $\left\{\mathbf{U}_{l}^{0}, \mathbf{V}_{l}^{0}\right\}=\left\{\mathbf{U}_{0}, \mathbf{V}_{0}\right\}$ over $\Omega_{l}^{h}$, one can write the finite element formulation of the substitution method as:

1. global analysis $\forall n \in\{1, \ldots, N\}$ :

$$
\begin{array}{ll}
\mathbf{M}_{g}{ }^{k} \mathbf{A}_{g}^{n+1}=\mathbf{F}_{\text {ext }}^{n+1}-\mathbf{F}_{g, \text { int }}^{n+1}+\mathbf{C}_{g}^{T k} \Delta \mathbf{F}_{g l}^{n+1} & \text { over } \Omega_{g}^{h} \\
\mathbf{U}_{g}^{n+1}=\overline{\mathbf{U}}^{n+1} & \text { along } \partial \Omega_{u}
\end{array}
$$

where $k$ represents the current iteration, $\mathbf{C}_{g}$ is a Boolean connectivity matrix which extracts the nodal values from the global domain $\Omega_{g}^{h}$ to the global interface $\Gamma_{g}^{h}$ (see appendix A), and $\Delta \mathbf{F}_{g l}$ is a corrective force which results from the local analysis (see Section 3.2) and is applied only to $\Omega_{g s}^{h}$ where the local analysis overlaps (see Figure 6); 
2. local analysis $\forall m \in\{1, \ldots, M \cdot N\}$ :

$$
\begin{array}{ll}
\mathbf{M}_{l}{ }^{k} \mathbf{A}_{l}^{m+1}=\mathbf{C}_{l}^{T k} \Lambda^{m+1}-\mathbf{F}_{l, \text { int }}^{m+1} & \text { over } \Omega_{l}^{h} \\
\mathbf{U}_{l}^{m+1}=\overline{\mathbf{U}}^{m+1} & \text { along } \partial \Omega_{u}
\end{array}
$$

where $\mathbf{C}_{l}$ is a Boolean connectivity matrix which extracts the nodal values from the local domain $\Omega_{l}^{h}$ to the local interface $\Gamma_{l}^{h}$ (see appendix A);

3. continuity of the velocities $\forall m \in\{1, \ldots, M \cdot N\}$ :

$$
\begin{aligned}
& \Pi_{h}^{1}\left(\mathbf{C}_{g}{ }^{k} \mathbf{V}_{g}^{m+1}\right)=\mathbf{C}_{l}{ }^{k} \mathbf{V}_{l}^{m+1} \quad \text { along } \Gamma_{l}^{h} \\
& { }^{k} \mathbf{V}_{g}^{m+1}=\Pi_{t}^{3}\left({ }^{k} \mathbf{V}_{g}^{n, n+1},{ }^{k} \mathbf{A}_{g}^{n, n+1}\right)
\end{aligned}
$$

where $\Pi_{h}^{1}(\cdot)$ is a linear spatial interpolation operator from the global interface $\Gamma_{g}^{h}$ to the local interface $\Gamma_{l}^{h}$ (see appendix A) and $\Pi_{t}^{3}(\cdot, \cdot)$ is a cubic temporal Hermitian interpolation operator (discussed in 3.3).

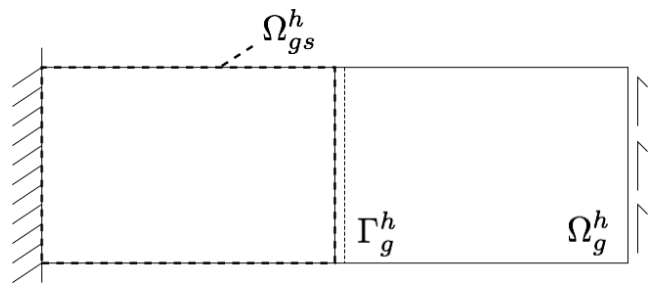

Fig. 6: Discretization of the overlapped part of the domain

From here on, the substitution finite element method will be particularized to the linear case.

\subsection{Meaning of the correction}

Here, the corrective nodal forces $\Delta \mathbf{F}_{g l}$ are determined as the difference between the global nodal forces $\mathbf{F}_{g}$ and the local nodal forces $\mathbf{F}_{l}$, such that:

$$
\begin{aligned}
& { }^{k} \mathbf{F}_{g}^{n+1}=\mathbf{C}_{g s}\left(\mathbf{M}_{g s}{ }^{k-1} \mathbf{A}_{g s}^{n+1}+\mathbf{F}_{g s, \text { int }}^{n+1}\right) \\
& { }^{k} \mathbf{F}_{l}^{n+1}=\mathbf{C}_{l}\left(\mathbf{M}_{l}{ }^{k} \mathbf{A}_{l}^{n+1}+{ }^{k} \mathbf{F}_{l, \text { int }}^{n+1}\right)={ }^{k} \Lambda^{n+1} \\
& { }^{k} \Delta \mathbf{F}_{g l}^{n+1}={ }^{k} \mathbf{F}_{g}^{n+1}-\tilde{\Pi}_{h}{ }^{1}\left({ }^{k} \mathbf{F}_{l}^{n+1}\right)
\end{aligned}
$$

where matrices $\mathbf{C}_{g s}, \mathbf{M}_{g s}$ and $\mathbf{F}_{g s \text {,int }}$ are defined taking into account only the overlapped part $\Omega_{g s}^{h}$ of the global domain (see Figure 6), and the local nodal forces $\mathbf{F}_{l}$ are projected through the global interface $\Gamma_{g}^{h}$ using the linear spatial operator $\tilde{\Pi}_{h}^{1}(\cdot)$.

Thus, the first equation of System (16) over $\Omega_{g}^{h}$ can be written by developing the corrective term $\Delta \mathbf{F}_{g l}$ :

$$
\mathbf{M}_{g}{ }^{k} \mathbf{A}_{g}^{n+1}=\mathbf{F}_{\mathrm{ext}}^{n+1}-\mathbf{F}_{g, \text { int }}^{n+1}+\mathbf{C}_{g}^{T}\left({ }^{k} \mathbf{F}_{g}^{n+1}-\tilde{\Pi}_{h}^{1}\left({ }^{k} \mathbf{F}_{l}^{n+1}\right)\right)
$$

which is equivalent to:

$$
\begin{aligned}
& \mathbf{M}_{g s}{ }^{k} \mathbf{A}_{g s}^{n+1}={ }^{k} \mathbf{F}_{g i}^{n+1}-\mathbf{F}_{g s, \text { int }}^{n+1}+{ }^{k} \mathbf{F}_{g}^{n+1}-\tilde{\Pi}_{h}{ }^{1}\left({ }^{k} \mathbf{F}_{l}^{n+1}\right) \\
& \text { over } \Omega_{g s}^{h} \text { and }{ }^{k} \mathbf{A}_{g}=\mathbf{0} \text { over } \Omega_{g}^{h} \backslash \Omega_{g s}^{h}
\end{aligned}
$$

where $\mathbf{F}_{g i}$ are the nodal reaction forces along global interface $\Gamma_{g}^{h}$ :

${ }^{k} \mathbf{F}_{g i}^{n+1}=\mathbf{C}_{g i}\left(\mathbf{M}_{g i}{ }^{k-1} \mathbf{A}_{g i}^{n+1}+\mathbf{F}_{g i, \text { int }}^{n+1}\right)$

and $\mathbf{M}_{g i}$ and $\mathbf{F}_{g i \text {,int }}$ are the mass matrix and the internal forces of the domain which is adjacent to the overlapped part $\Omega_{g s}^{h}$.

After dividing the global acceleration term into a precomputed part and a corrective part, Equation (21) becomes:

$$
\begin{array}{r}
\mathbf{M}_{g s}\left(\mathbf{A}_{g s, \text { prec }}^{n+1}+{ }^{k} \mathbf{A}_{g s, \text { corr }}^{n+1}\right)={ }^{k} \mathbf{F}_{g i}^{n+1}-\mathbf{F}_{g s, \text { int }}^{n+1}+ \\
+{ }^{k} \mathbf{F}_{g}^{n+1}-\tilde{\Pi}_{h}^{1}\left({ }^{k} \mathbf{F}_{l}^{n+1}\right)
\end{array}
$$

Then, using the definition of ${ }^{k} \mathbf{F}_{g}^{n+1}$ (the first equation of System (19)):

$\mathbf{M}_{g s}\left({ }^{k} \mathbf{A}_{g s, \text { corr }}^{n+1}-{ }^{k-1} \mathbf{A}_{g s, \text { corr }}^{n+1}\right)={ }^{k} \mathbf{F}_{g i}^{n+1}-\tilde{\Pi}_{h}^{1}\left({ }^{k} \mathbf{F}_{l}^{n+1}\right)$

After imposing the continuity of velocities along interface $\Gamma_{l}^{h}$, one iterates until the equilibrium between the nodal reaction forces $\mathbf{F}_{g i}$ and the local nodal forces $\mathbf{F}_{l}$ has been reached.

Then, from Equation (24):

${ }^{k, k-1} \Delta \mathbf{A}_{g s, \text { corr }}^{n+1} \rightarrow \mathbf{0} \quad \Leftrightarrow \quad \tilde{\Pi}_{h}^{1}\left({ }^{k} \mathbf{F}_{l}^{n+1}\right) \rightarrow{ }^{k} \mathbf{F}_{g i}^{n+1}$

The measure of the convergence of the equilibrium of the nodal reaction forces $\mathbf{F}_{g i}$ and the local nodal forces $\mathbf{F}_{l}$ is:

$e=\frac{\left\|{ }^{k} \mathbf{F}_{g i}^{n+1}-\tilde{\Pi}_{h}^{1}\left({ }^{k} \mathbf{F}_{l}^{n+1}\right)\right\|_{L^{2}\left(\Gamma_{g}^{h}\right)}}{\left\|\mathbf{F}_{\mathrm{ext}}^{n+1}\right\|_{L^{2}\left(\partial \Omega_{f}\right)}}$

where $\|\cdot\|_{L^{2}(\cdot)}$ represents an $L^{2}$ norm which, in this case, is calculated along interfaces $\Gamma_{g}^{h}$ and $\partial \Omega_{f}$.

3.3 Cubic Hermitian temporal interpolation of the global velocities

Equation (18) states the velocity constraint between the two analyses along interface $\Gamma_{l}^{h}$. Here, this constraint is satisfied along the refined interface $\Gamma_{l}^{h}$ at the end of each refined time step $\Delta t_{l}$.

The global velocities are calculated along the global interface $\Gamma_{l}^{h}$ and at the end of each coarse time step $\Delta t_{g}$. This requires two approximations: a linear spatial interpolation $\Pi_{h}^{1}\left(\mathbf{C}_{g} \mathbf{V}_{g}^{m+1}\right)$ and a cubic temporal Hermitian interpolation $\mathbf{V}_{g}^{m+1}=\Pi_{t}^{3}\left(\mathbf{V}_{g}^{n, n+1}, \mathbf{A}_{g}^{n, n+1}\right)$, so that:

$$
\begin{aligned}
\mathbf{V}_{g}^{m+1}=(1 & \left.-3 s^{2}+2 s^{3}\right) \mathbf{V}_{g}^{n}+\Delta t_{g}\left(s-2 s^{2}+s^{3}\right) \mathbf{A}_{g}^{n}+ \\
& +\left(3 s^{2}-2 s^{3}\right) \mathbf{V}_{g}^{n+1}+\Delta t_{g}\left(-s^{2}+s^{3}\right) \mathbf{A}_{g}^{n+1}
\end{aligned}
$$


with $s=(m+1) / M$.

A simple linear interpolation $\Pi_{t}^{1}(\cdot)$ would not be compatible with the explicit time integration scheme and would lead to numerical instability of the local accelerations at the interface. Indeed, with a linear interpolation $\Pi_{t}^{1}(\cdot)$, one would have at each local time step:

$\mathbf{C}_{l}\left(\mathbf{V}_{l}^{m+1}-\mathbf{V}_{l}^{m}\right)=\mathbf{C}_{g}\left(\mathbf{V}_{g}^{n+1}-\mathbf{V}_{g}^{n}\right) / M$

which is constant throughout the local analysis between times $t^{n}$ and $t^{n+1}$.

Then, the Newmark scheme can be rewritten by making a change of variables between Equations (8) and (9), leading to the resolution of (9) in terms of velocities. Thus, from (8):

$\frac{\Delta t}{2}\left(\mathbf{A}^{n+1}+\mathbf{A}^{n}\right)=\mathbf{V}^{n+1}-\mathbf{V}^{n}$

and Equation (28) becomes:

$\frac{\Delta t_{l}}{2} \mathbf{C}_{l}\left(\mathbf{A}_{l}^{m+1}+\mathbf{A}_{l}^{m}\right)=\frac{\Delta t_{g}}{2 M} \mathbf{C}_{g}\left(\mathbf{A}_{g}^{n+1}+\mathbf{A}_{g}^{n}\right)$

which, from the definition of the global time step $\Delta t_{g}=$ $M \Delta t_{l}$, is equivalent to:

$\mathbf{C}_{l}\left(\mathbf{A}_{l}^{m+1}+\mathbf{A}_{l}^{m}\right)=\mathbf{C}_{g}\left(\mathbf{A}_{g}^{n+1}+\mathbf{A}_{g}^{n}\right)$

Equation (31) is satisfied at each local time step of the entire analysis. Therefore, with the homogeneous initial conditions $\mathbf{A}_{l}^{0}=\mathbf{0}$, if $M$ is even, $\mathbf{A}_{l}^{m+1}$ oscillates between $\mathbf{A}_{g}^{n}$ and $\mathbf{A}_{g}^{n+1} \forall m=1, \ldots, M$; if $M$ is odd, $\mathbf{A}_{l}^{m+1}$ oscillates between $\mathbf{0}$ and $\left(\mathbf{A}_{g}^{n}+\mathbf{A}_{g}^{n+1}\right) \forall m=1, \ldots, M$.

So, the approximations of the global velocities $\mathbf{V}_{g}^{m+1}$ has been enriched using a cubic Hermitian interpolation, including additional conditions on the global accelerations $\mathbf{A}_{g}^{n}$ and $\mathbf{A}_{g}^{n+1}$. Thus, Equation (28) is not satisfied during the local analysis.

\subsection{Definition of the Lagrange multipliers}

Lagrange multipliers are used in order to ensure that Equation (18) is satisfied along the local interface $\Gamma_{l}^{h}$ at the end of each refined time step $\Delta t_{l}$ of the local analysis.

If one develops the Newmark scheme for the local velocities $\mathbf{V}_{l}^{m+1}$ as in Equation (9), Equation (18) becomes:

$\Pi_{h}^{1}\left(\mathbf{C}_{g} \mathbf{V}_{g}^{m+1}\right)=\mathbf{C}_{l} \mathbf{V}_{l}^{m}+\mathbf{C}_{l} \frac{\Delta t_{l}}{2}\left(\mathbf{A}_{l}^{m}+\mathbf{A}_{l}^{m+1}\right)$

Then, separating the free and link solutions as in the domain decomposition approach [9] such that:

$\mathbf{A}_{l}^{m+1}=\mathbf{A}_{l, \text { free }}^{m+1}+\mathbf{A}_{l, \text { link }}^{m+1}$

and defining the Lagrange multipliers $\Lambda^{m+1}$ as:

$\mathbf{M}_{l} \mathbf{A}_{l, \text { link }}^{m+1}=\mathbf{C}_{l}^{T} \Lambda^{m+1}$ the Equation (32) writes:

$\Pi_{h}^{1}\left(\mathbf{C}_{g} \mathbf{V}_{g}^{m+1}\right)=\mathbf{C}_{l} \mathbf{V}_{l}^{m}+\mathbf{C}_{l} \frac{\Delta t_{l}}{2} \mathbf{A}_{l, \text { link }}^{m+1}+\mathbf{C}_{l} \frac{\Delta t_{l}}{2}\left(\mathbf{A}_{l}^{m}+\mathbf{A}_{l, \text { free }}^{m+1}\right)$

and, using Equation (34), Equation (35) becomes:

$$
\begin{aligned}
\Pi_{h}^{1}\left(\mathbf{C}_{g} \mathbf{V}_{g}^{m+1}\right)=\mathbf{C}_{l} \mathbf{V}_{l}^{m}+ & \frac{\Delta t_{l}}{2} \mathbf{C}_{l} \mathbf{M}_{l}^{-1} \mathbf{C}_{l}^{T} \Lambda^{m+1}+ \\
& +\mathbf{C}_{l} \frac{\Delta t_{l}}{2}\left(\mathbf{A}_{l}^{m}+\mathbf{A}_{l, \text { free }}^{m+1}\right)
\end{aligned}
$$

which is equivalent to:

$$
\begin{array}{r}
\left(\frac{\Delta t_{l}}{2} \mathbf{C}_{l} \mathbf{M}_{l}^{-1} \mathbf{C}_{l}^{T}\right) \Lambda^{m+1}=\Pi_{h}^{1}\left(\mathbf{C}_{g} \mathbf{V}_{g}^{m+1}\right)-\mathbf{C}_{l} \mathbf{V}_{l}^{m}+ \\
-\mathbf{C}_{l} \frac{\Delta t_{l}}{2}\left(\mathbf{A}_{l}^{m}+\mathbf{A}_{l, \text { free }}^{m+1}\right)
\end{array}
$$

In the following, we denote $\mathbf{H}_{l}$ the local interface condensation matrix:

$\mathbf{H}_{l}=\frac{\Delta t_{l}}{2} \mathbf{C}_{l} \mathbf{M}_{l}^{-1} \mathbf{C}_{l}^{T}$

$\mathbf{H}_{l}$ is a diagonal matrix like $\mathbf{M}_{l}$ and, in the linear case, is calculated only once at the beginning of the analysis. This can be extended to the nonlinear case by analogy with the domain decomposition extension described in [20].

Remark 1 For domain decomposition approaches, in order to save computing time, it is possible to impose continuity of the velocities (Equation (18)) along global interface $\Gamma_{g}^{h}$ : in this case, the Lagrange multipliers are calculated at the global interface nodes, the connectivity matrix $\mathbf{C}_{l}$ refers directly to global interface $\Gamma_{g}^{h}$ and there is no need to interpolate $\Pi_{h}^{1}(\cdot)$ linearly from global interface $\Gamma_{g}^{h}$ to local interface $\Gamma_{l}^{h}$. Equation (18) becomes:

$\mathbf{C}_{l} \mathbf{V}_{l}^{m+1}=\mathbf{C}_{g} \mathbf{V}_{g}^{m+1} \quad$ along $\Gamma_{g}^{h}$

Furthermore, the calculation of the correction term $\Delta \mathbf{F}_{g l}$ of the global analysis does not require the linear spatial projection of the local nodal forces $\mathbf{F}_{l}$ : then, the third term of (19) writes:

${ }^{k} \Delta \mathbf{F}_{g l}^{n+1}={ }^{k} \mathbf{F}_{g}^{n+1}-{ }^{k} \mathbf{F}_{l}^{n+1}$

\subsection{Results}

Problem (1) was solved using the following data:

- density $\rho=7,800 \mathrm{~kg} / \mathrm{m}^{3}$;

- Young's modulus $E=210 \cdot 10^{9} \mathrm{~Pa}$;

- Poisson's ratio $v=0.3$;

- length (see Figure 1) $l=30 \mathrm{~m}$;

- height (see Figure 1) $h=10 m$;

- initial time $t_{0}=0 \mathrm{~s}$; 
- final time $t_{\text {end }}=0.5 \mathrm{~s}$;

- shearing load $\bar{f}=3 \cdot 10^{6} \mathrm{~Pa}$ applied to a portion of $\partial \Omega_{f}$.

The spatial and temporal discretizations are shown in Figures 4 and 5 .

The critical time step was estimated using Equations (5) and (6), leading, for the global analysis, to $\Delta t_{\mathrm{cr}}=1.66 \cdot 10^{-3}$ $s$. We focused on the results at two representative points of the domain: internal point A and external point B (see Figure 7.)

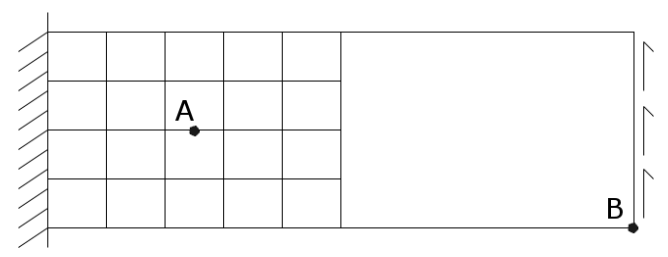

Fig. 7: The points of interest chosen: point $\mathrm{A}$ in the local zone and point $B$ at the end of the structure

The substitution finite element method was validated by comparison with the dual domain decomposition finite element method presented in [28] using the equivalent discretization of Figure 3.

\subsubsection{Comparison and influence of the time step size}

First, the analysis was carried out using an efficient time step chosen to be almost equal to the critical time step $\Delta t_{c r}$ :

- $\Delta t_{g}=1.2 \cdot 10^{-3} s$ for the global analysis;

$-\Delta t_{l}=0.3 \cdot 10^{-3} s$ for the local analysis.

The corresponding numerical results are shown in Figures 8 and 9. With this temporal discretization, the accelerations matched perfectly from the beginning of the analysis to $t=0.05 \mathrm{~s}$, after which some divergences became visible.

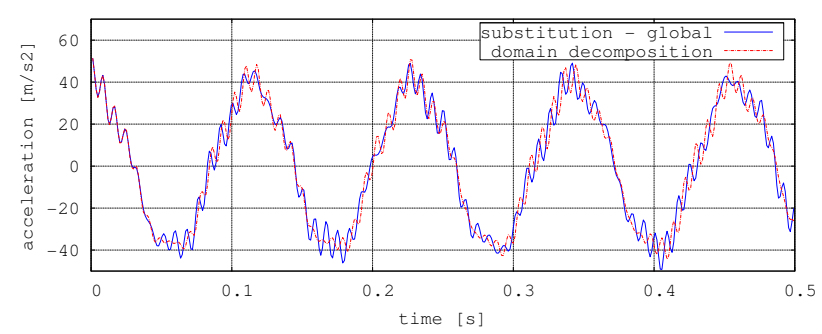

Fig. 8: The vertical accelerations at point B: comparison between substitution and domain decomposition

This indicates that a temporal discretization error accumulated during the analysis, causing the progressive divergence of the two solutions.

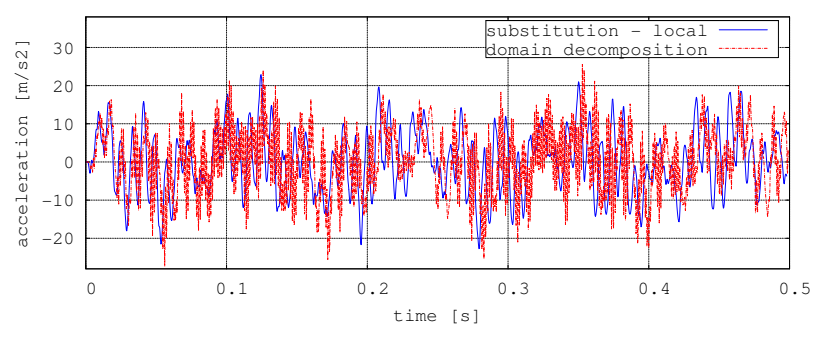

Fig. 9: The vertical accelerations at point A: comparison between substitution and domain decomposition

Then, the same analyses as in Figures 8 and 9 were carried out, this time using smaller time steps in order to verify the convergence of the two methods toward the same solution. The time steps used were:

- $\Delta t_{g}=0.4 \cdot 10^{-3} s$ for the global analysis;

- $\Delta t_{l}=0.1 \cdot 10^{-3} s$ for the local analysis.

The corresponding numerical results are shown in Figures 10 and 11. The two results matched perfectly for the entire duration of the analysis.

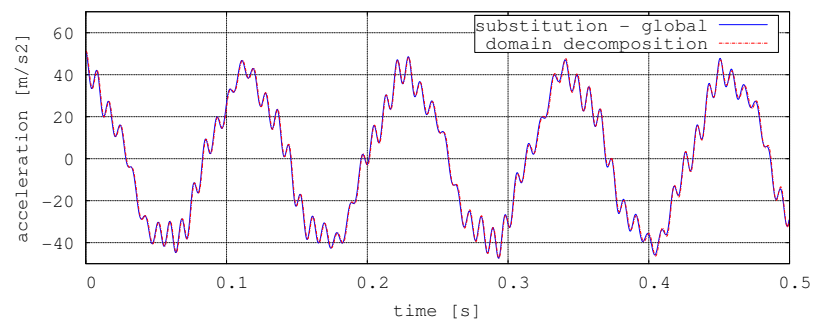

Fig. 10: The vertical accelerations at point B: comparison between substitution and domain decomposition

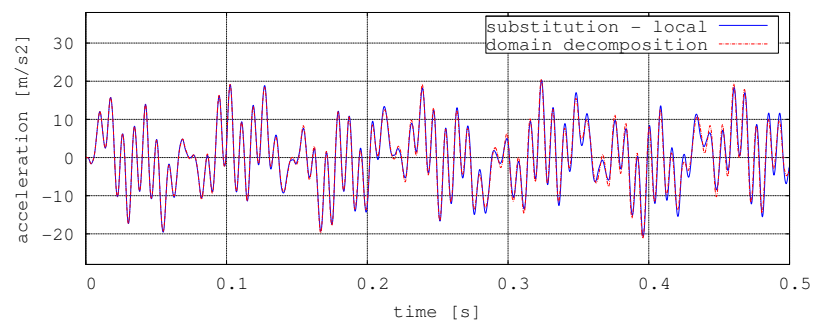

Fig. 11: The vertical accelerations at point A: comparison between substitution and domain decomposition

This shows that if one refines the temporal discretization the substitution finite element method and the domain decomposition finite element method converge toward the same solution. 


\subsubsection{Accuracy study}

The formulations of the domain decomposition and substitution methods are different, but we have not yet addressed the question of which of the two is more accurate. Now, in order to find out which method converges faster toward the exact solution, let us compare the results of the domain decomposition finite element method [28] and the substitution finite element method with cubic temporal Hermitian interpolation of the global velocities when one refines the time steps. We carried out three analyses:

- the first one with a coarse time step $\Delta t_{g}=0.0012 \mathrm{~s}$;

- the second one with a coarse time step $\Delta t_{g}=0.0008 \mathrm{~s}$;

- the third one with a coarse time step $\Delta t_{g}=0.0004 \mathrm{~s}$.

Figure 12 compares the accuracy of the two methods by monitoring the vertical accelerations at point B (see Figure 7) at the beginning of the analysis until $t=0.1 \mathrm{~s}$. Figure 12a shows the convergence of the domain decomposition finite element method toward the exact solution while Figure $12 \mathrm{~b}$ shows the convergence of the substitution finite element method toward the exact solution.

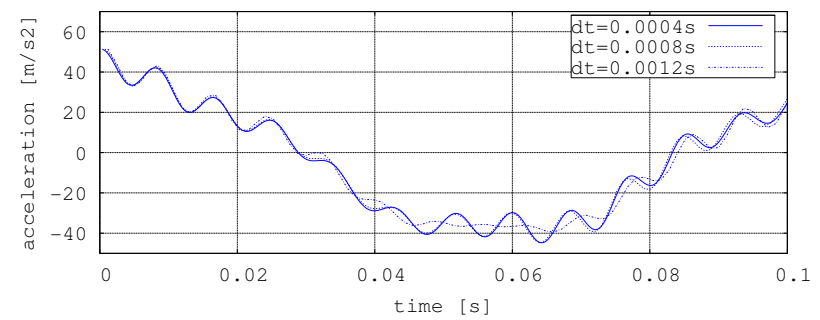

(a) Domain decomposition finite element method

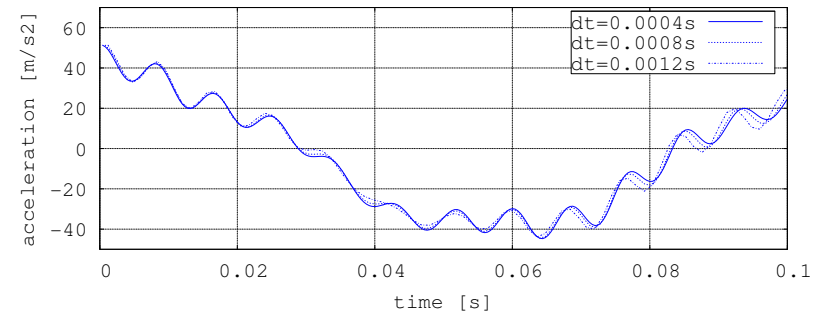

(b) Substitution finite element method with Hermitian interpolation

Fig. 12: The vertical acceleration at point B: accuracy study when refining the temporal discretization

The substitution finite element method converged toward the exact solution faster, but also accumulated a larger error as a function of time.

\subsubsection{Convergence study of the iterative coupling}

Figure 13 shows the decreasing evolution of the convergence measure $e$ defined by (26) at time $t_{g}=0.25 \mathrm{~s}$ as a function of the number of iterations up to $n_{\mathrm{it}}=20$. The level of accuracy is considered to be reasonable when $e$ becomes smaller than $0.01 \%$.

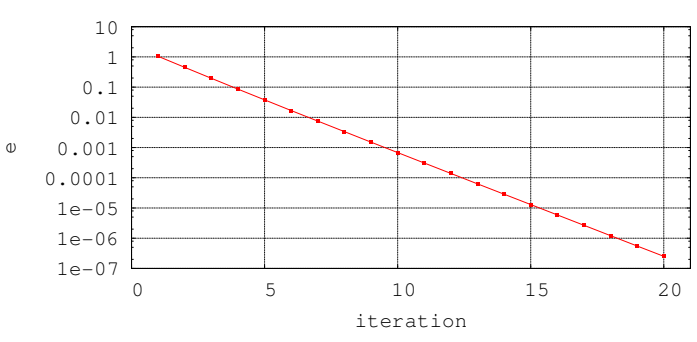

Fig. 13: The convergence at $t_{g}=0.25 \mathrm{~s}$

The iterations are carried out until $e$ becomes less than a convergence tolerance $\bar{e}$ representing the acceptable imbalance between the local nodal forces and the global reaction forces. In our case, taking $\bar{e}$ equal to $0.01 \%$, the required number of iterations varied between 4 and 11 throughout the duration of the analysis (see Figure 14.)

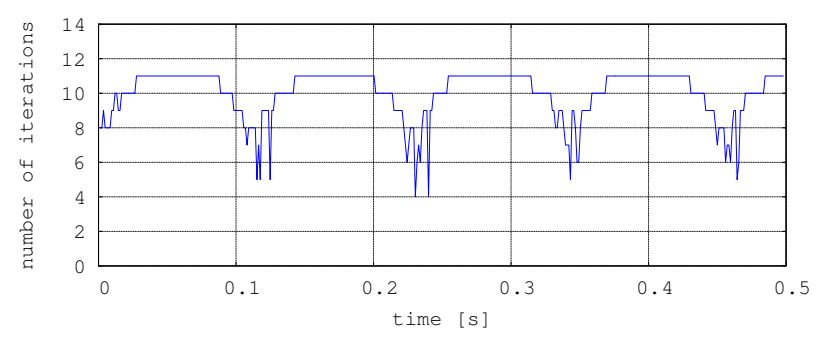

Fig. 14: The number of iterations during the simulation for a convergence tolerance $\bar{e}=0.01 \%$

\subsubsection{Stability study}

As underlined by [31] and [27], controlling independent variables is insufficient to guarantee the robustness of a coupling method. We used the energy method [25] to test the stability and robustness of the substitution finite element method.

In the domain decomposition method, the Lagrange multipliers are applied to the interfaces between the subdomains and equilibrium is always satisfied. Conversely, in the substitution method, the Lagrange multipliers are applied only to the interface of the local analysis, so the equilibrium with the global reaction forces along global interface $\Gamma_{g}^{h}$ is reached iteratively. An important consequence is that the equilibrium is always guaranteed only within an error range which must be controlled by the convergence tolerance $\bar{e}$. Therefore, we calculated the variations of the power terms associated with the work of the Lagrange multipliers and the work of the 
global reactions. The sum of these terms is shown in the following figures: in Figure 15, it is compared to the numerical dissipation of the domain decomposition approach with linearly interpolated velocities [10]; in Figures 16, it is shown with different values of the convergence tolerance $\bar{e}$, where the residual of the power variation is normalized by a target value $\dot{P}_{100 \%}=10^{9} \dot{W}$, a term linked to the work of the external load.

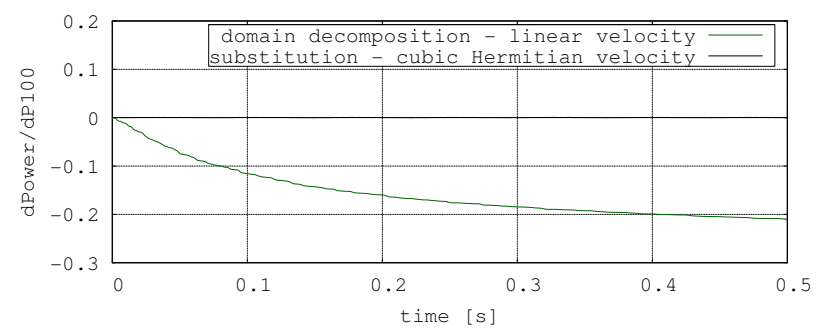

Fig. 15: Comparison between the domain decomposition method with linear interpolation of velocities and the substitution method with cubic Hermitian interpolation of velocities

The results shown in Figure 16 indicate that when the convergence tolerance $\bar{e}$ was reduced the residual power variation did not decrease, but converged toward values which oscillated around $0.03 \%$ of $\dot{P}_{100 \%}$. These values are negligible for this test case, but they could compromise the accuracy or the stability of analyses performed on larger structures in which the interface would involve more extensive zones.

\section{An energy preserving substitution method}

By analogy with the domain decomposition study, we propose an alternative method using the power conservation formulation. We introduce a new definition of the Lagrange multipliers inspired by [31] and [28]: now, continuity of the velocities is enforced only at the coarse time steps, and the Lagrange multipliers are calculated taking into account the sequences of the refined time steps, and then are linearly interpolated in time.

\subsection{Definition of the new Lagrange multipliers}

The changes in the substitution method affect primarily Equations (17) and (18). Specifically, the local analysis (Equation (17)) becomes:

$$
\begin{array}{ll}
\mathbf{M}_{l}{ }^{k} \mathbf{A}_{l}^{m+1}=\mathbf{F}_{l, \text { ext }}^{m+1}-\mathbf{F}_{l, \text { int }}^{m+1}+\mathbf{C}_{l}^{T} \Pi_{t}^{1}\left({ }^{k} \Lambda^{n, n+1}\right) & \text { over } \Omega_{l}^{h} \\
\mathbf{U}_{l}^{m+1}=\overline{\mathbf{U}}^{m+1} & \text { along } \partial \Omega_{u}
\end{array}
$$

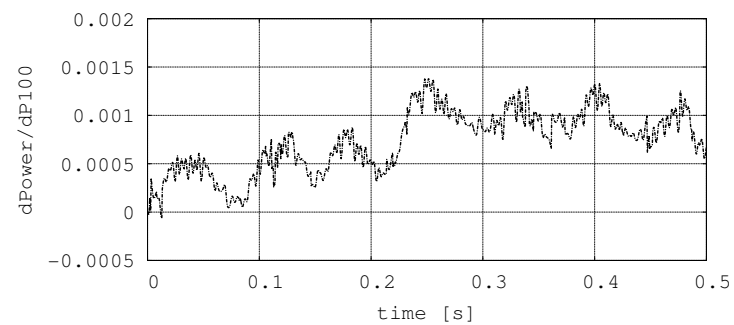

(a) convergence tolerance $\bar{e}=0.1 \%$

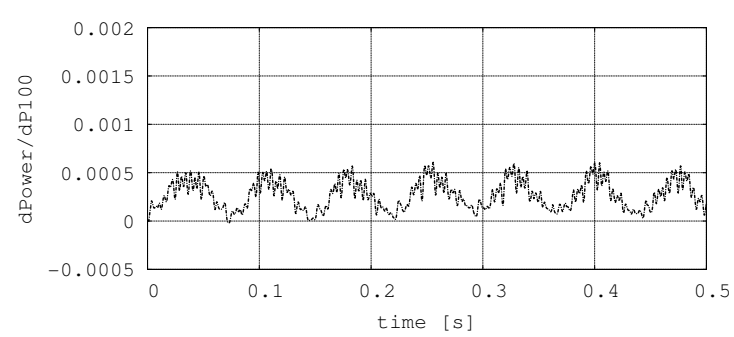

(b) convergence tolerance $\bar{e}=0.01 \%$

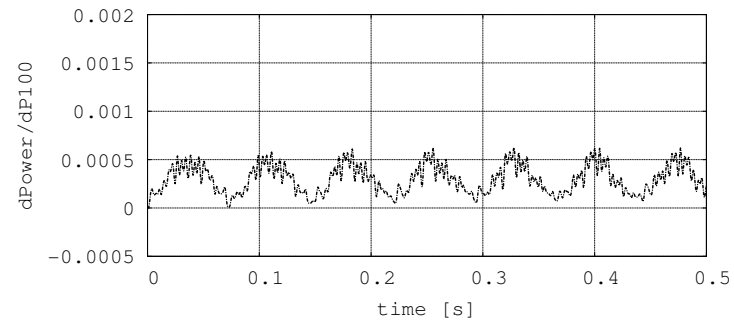

(c) convergence tolerance $\bar{e}=0.001 \%$

Fig. 16: The residual power variation of the coupling using the cubic Hermitian temporal interpolation for the global velocities

and the expression of the continuity of velocities (Equation (18)) becomes:

$$
\Pi_{h}^{1}\left(\mathbf{C}_{g}{ }^{k} \mathbf{V}_{g}^{n+1}\right)=\mathbf{C}_{l}^{k} \mathbf{V}_{l}^{n+1} \text { along } \Gamma_{l}^{h}
$$

where ${ }^{k} \mathbf{V}_{l}^{n+1}$ denotes the local velocities at coarse time $t^{n+1}$.

By analogy with [31] and [28], Systems (41) and (42) can be written in monolithic form for each coarse time step $\Delta t_{g}$, leading to:

$$
\forall n=0, \ldots, N
$$

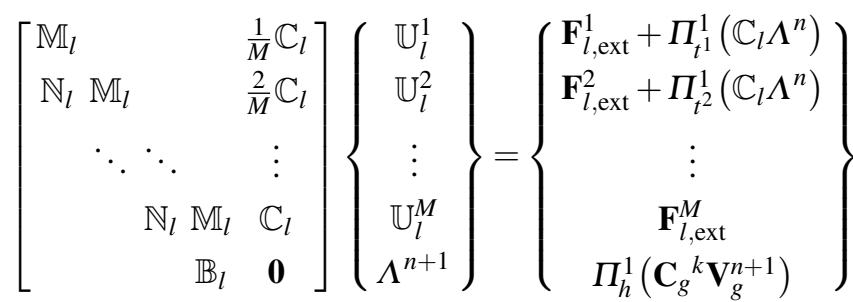


where:

$$
\begin{gathered}
\mathbb{M}=\left[\begin{array}{ccc}
\mathbf{M} & \mathbf{0} & \mathbf{0} \\
-\frac{\Delta t}{2} \mathbf{I} & \mathbf{I} & \mathbf{0} \\
\mathbf{0} & \mathbf{0} & \mathbf{I}
\end{array}\right] \quad \mathbb{N}=\left[\begin{array}{ccc}
\frac{\Delta t^{2}}{2} \mathbf{K} & \Delta t \mathbf{K} & \mathbf{K} \\
-\frac{\Delta t}{2} \mathbf{I} & -\mathbf{I} & \mathbf{0} \\
-\frac{\Delta t^{2}}{2} \mathbf{I} & -\Delta t \mathbf{I} & -\mathbf{I}
\end{array}\right] \\
\mathbb{U}=\left\{\begin{array}{l}
\mathbf{A} \\
\mathbf{V} \\
\mathbf{U}
\end{array}\right\} \quad \mathbb{C}=\left[\begin{array}{c}
\mathbf{C}^{T} \\
\mathbf{0} \\
\mathbf{0}
\end{array}\right] \quad \mathbb{B}=\left[\begin{array}{lll}
\mathbf{0} & \mathbf{C} & \mathbf{0}
\end{array}\right]
\end{gathered}
$$

where $\mathbf{K}$ is the stiffness matrix and with the assumption, for the sake of simplicity, that $\mathbb{U}_{l}^{0}=\mathbf{0}$. I denotes the identity matrix.

The monolithic matrix System (43) can be rewritten as: $\forall n=0, \ldots, N$

$$
\left[\begin{array}{ll}
\mathrm{M} & \mathrm{C} \\
\mathrm{B} & \mathbf{0}
\end{array}\right]\left\{\begin{array}{l}
\mathrm{U}^{n+1} \\
\Lambda^{n+1}
\end{array}\right\}=\left\{\begin{array}{c}
\mathrm{F}+\Pi_{t}^{1}\left(\Lambda^{n}\right) \\
\mathbf{0}
\end{array}\right\}
$$

where $\mathrm{M}$ is a lower triangular matrix composed of matrices $\mathbb{M}$ and $\mathbb{N} ; C$ is the matrix containing the linear interpolation of matrices $\mathbb{C}$; $B$ is a Boolean matrix which consists of matrix $\mathbb{B}$ plus some zeros; $U$ is the displacement-velocityacceleration vector and $\mathrm{F}$ is the force vector.

Then, the free-link approach is used to divide the variable vector $U$ into $U_{\text {free }}+U_{\text {link }}$ and where Lagrange multipliers are defined as $\mathrm{MU}_{\text {link }}=\mathrm{C} \Lambda^{n+1}$. So, the System (44) can be solved in three steps:

1. resolution of the free problem:

$$
\mathrm{MU}_{\text {free }}^{n+1}=\mathrm{F}+\Pi_{t}^{1}\left(\Lambda^{n}\right)
$$

2. calculation of the Lagrange multipliers with

$$
\mathrm{M} \mathbb{Y}=\mathrm{C}
$$

and

$$
\mathrm{B} \mathbb{Y} \Lambda^{n+1}=\mathrm{BU}_{\text {free }}^{n+1}
$$

where $\mathrm{B} \mathbb{Y}=\mathbf{H}_{l}$ is the condensation matrix and $\mathrm{BU}_{\text {free }}^{n+1}=$ $\mathbf{C}_{1} \mathbf{V}_{1, \text { free }}^{M}+\mathbf{C}_{2} \mathbf{V}_{2, \text { free }}^{n+1}$.

3. resolution of the link problem:

$$
\mathrm{U}_{\text {link }}^{n+1}=\mathbb{Y} \Lambda^{n+1}
$$

It is important to note that, in this case, all the variables of displacement, velocity and acceleration are decomposed in free variables and link variables (not only the acceleration as in Section 3).

With this free-link variable separation, the condensation matrix $\mathbf{H}_{l}$ has nonzero square blocks on the diagonal and the local analysis is carried out using two time loops. Here, Remark 1 becomes important because the size of the nonzero square blocks affects the computing time and requires a matrix solver. In the linear case, like for the first algorithm, the condensation matrix $\mathbf{H}_{l}$ is calculated only once.

\subsection{Results}

We considered the same two-dimensional problem as in 3.5.

\subsubsection{Comparison and influence of the time step size}

The analysis was carried out using the same efficient time step as in Subsection 3.5.1:

- $\Delta t_{g}=1.2 \cdot 10^{-3} s$ for the global analysis;

$-\Delta t_{l}=0.3 \cdot 10^{-3} s$ for the local analysis.

The corresponding numerical results are shown in Figures 17 and 18

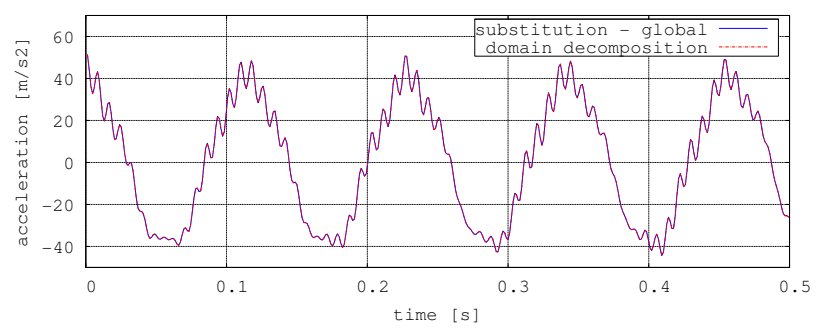

Fig. 17: The vertical accelerations at point B: comparison between improved substitution and domain decomposition

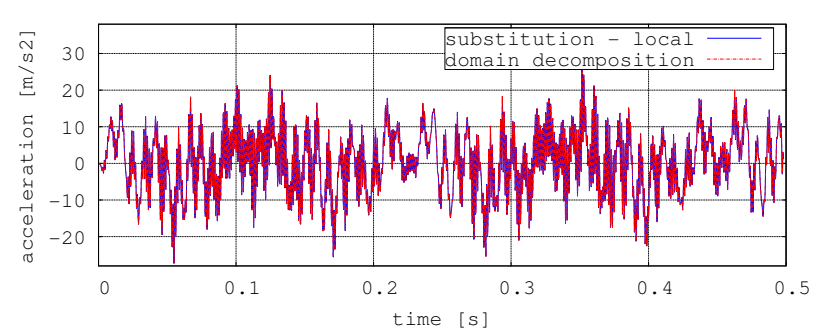

Fig. 18: The vertical accelerations at point A: comparison between improved substitution and domain decomposition

With this temporal discretization, too, the accelerations matched perfectly throughout the duration of the analysis: actually, the same approach regarding the definition of the Lagrange multipliers led to the same solution with both methods, so it was unnecessary to apply a time step refinement in order to verify the convergence toward the same solution.

\subsubsection{Stability study}

As in Section 3.5.4, the energy method [25] was used to verify the stability of the coupling scheme. The results are shown in Figure 19.

The residual power variation was found to be proportional to the convergence tolerance $\bar{e}$ from $\bar{e}=0.1 \%$ to $\bar{e}=$ 


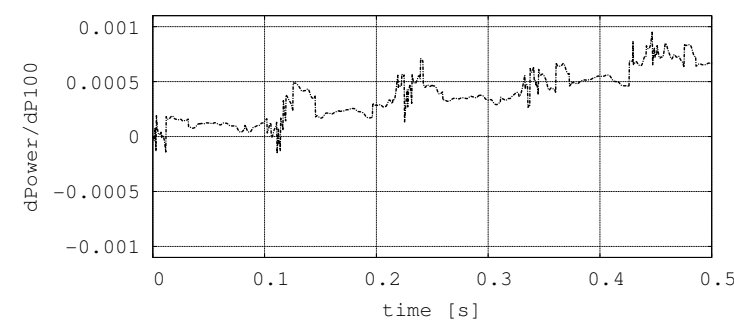

(a) convergence tolerance $\bar{e}=0.1 \%$

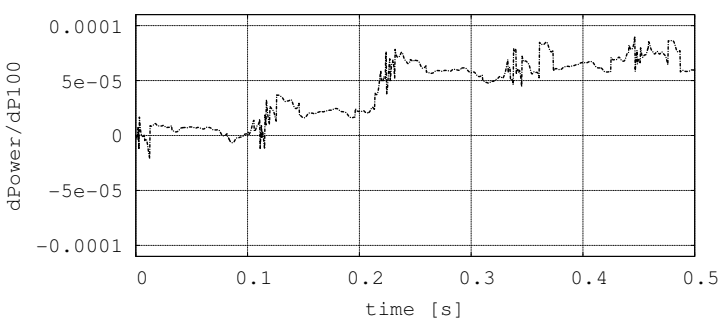

(b) convergence tolerance $\bar{e}=0.01 \%$

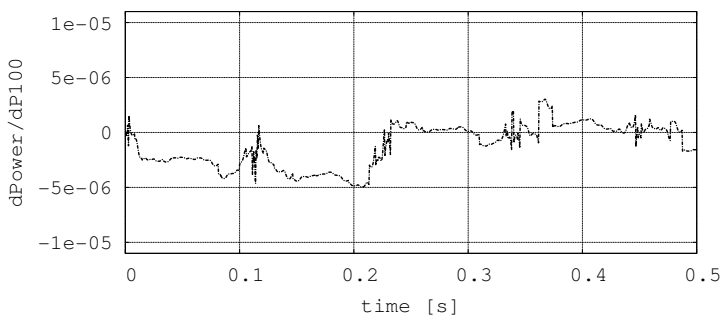

(c) convergence tolerance $\bar{e}=0.001 \%$

Fig. 19: The residual power variation of the coupling using the new algorithm for calculating the Lagrange multipliers

$0.001 \%$, which shows that the stability, accuracy and robustness are preserved and controlled by parameter $\bar{e}$.

\section{Stabilization}

So, both the results in Figures 16 and 19 stated that the residual power variation decreased consequently to the convergence tolerance decrease: in particular, the residual power variation converged to an oscillatory curve in Figures 16 and to zero in Figures 19.

This residual variation of power could compromise the stability of the coupling scheme and was due to the residual force of the iterative coupling that arbitrarily cumulated or subtracted power variation at each global time step.

Therefore, a stabilizer force $\mathbf{R}_{g l}^{n+1}$ has been applied to the global momentum balance (first equation of System (16)) for both the methodologies described in Sections 3 and 4 at the end of each global time step, in order to cover the residual gap between the two analyses. In this way, the cumula- tion or the subtraction of the residual variation of power is avoided.

Now, the global momentum balance equation writes:

$\mathbf{M}_{g}{ }^{k} \mathbf{A}_{g}^{n+1}=\mathbf{F}_{\mathrm{ext}}^{n+1}-\mathbf{F}_{g, \text { int }}^{n+1}+\mathbf{C}_{g}^{T}\left({ }^{k} \Delta \mathbf{F}_{g l}^{n+1}+\mathbf{R}_{g l}^{n+1}\right)$

The stabilizer force is equal to the residual force at the final iteration $K$ when $e<\bar{e}$ (see Equation (26)) and writes:

$\mathbf{R}_{g l}^{n+1}=\tilde{\Pi}_{h}^{1}\left({ }^{K} \mathbf{F}_{l}^{n+1}\right)-{ }^{K} \mathbf{F}_{g i}^{n+1}$

Furthermore, the Equation (49) is solved in three steps, so that the acceleration is decomposed as:

${ }^{k} \mathbf{A}_{g}^{n+1}=\mathbf{A}_{g, \text { prec }}^{n+1}+{ }^{k} \mathbf{A}_{g, \text { corr }}^{n+1}+\mathbf{A}_{g, \text { stab }}^{n+1}$

and the final procedure writes:

1. pre-computation step for computing $\mathbf{A}_{g, p r e c}^{n+1}$ :

$$
\mathbf{M}_{g} \mathbf{A}_{g, \text { prec }}^{n+1}=\mathbf{F}_{\mathrm{ext}}^{n+1}-\mathbf{F}_{g, \text { int }}^{n+1}
$$

2. correction step with the iterative local analysis for computing ${ }^{k} \mathbf{A}_{g, \text { corr }}^{n+1}$ :

$$
\mathbf{M}_{g}{ }^{k} \mathbf{A}_{g, \mathrm{corr}}^{n+1}=\mathbf{C}_{g}^{T k} \Delta \mathbf{F}_{g l, \mathrm{corr}}^{n+1}
$$

3. stabilization step for computing $\mathbf{A}_{g, s t a b}^{n+1}$ :

$$
\mathbf{M}_{g} \mathbf{A}_{g, \mathrm{stab}}^{n+1}=\mathbf{C}_{g}^{T} \mathbf{R}_{g l}^{n+1}
$$

The same stability study described in Subsections 3.5.4 and 4.2.2 was performed in order to verify the new stabilization procedure, at first for the algorithm introduced in Section 3, then for the new algorithm described in Section 4.

Figure 20 shows the variation of power related to the coupling carried out with:

- $\Delta t_{g}=0.4 \cdot 10^{-3} s$ for the global analysis;

$-\Delta t_{l}=0.1 \cdot 10^{-3} s$ for the local analysis;

$-\bar{e}=1 \%$ as convergence tolerance of the coupling.

The results in Figure 20 confirm that the stabilizer force completely removes the residual variation of power due to the cumulated equilibrium error and lets the utilization of a convergence tolerance more performant (here, $\bar{e}=1 \%$ ): the oscillating curve in Figure 20a is comparable to the oscillating curve in Figure 16c obtained with a convergence tolerance 1000 times smaller. Then, for the same analysis, Figure 20b emphasizes the gain in terms of stability using the stabilizer force: two analyses with the same performant convergence tolerance are accomplished with and without the stabilizer force. In addition, Figure 20c shows the same analysis with a bigger number of time steps: 10,000 global time steps and 40,000 local time steps.

Then, the same stabilization technique was applied to the new algorithm introduced in Section 4, based on an energy preserving formulation of the continuity of velocities at the interface. Coherently with the results discussed above, the 


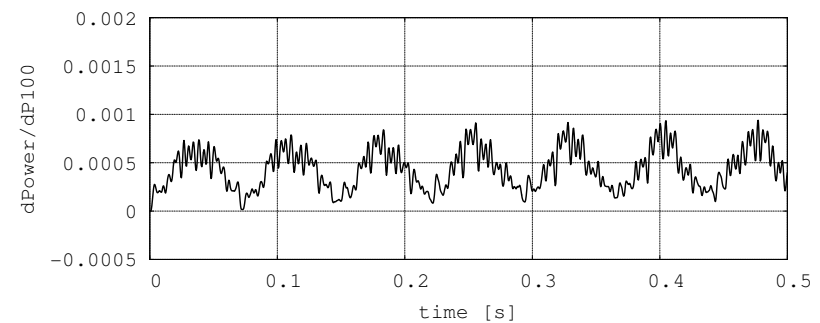

(a) analysis with the stabilizer force

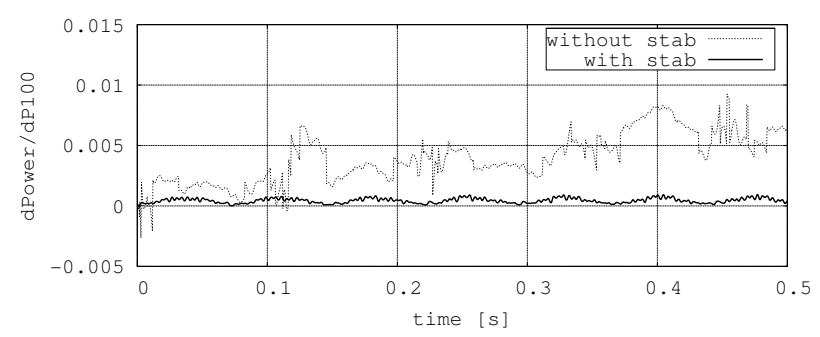

(b) comparison with or without the stabilizer force

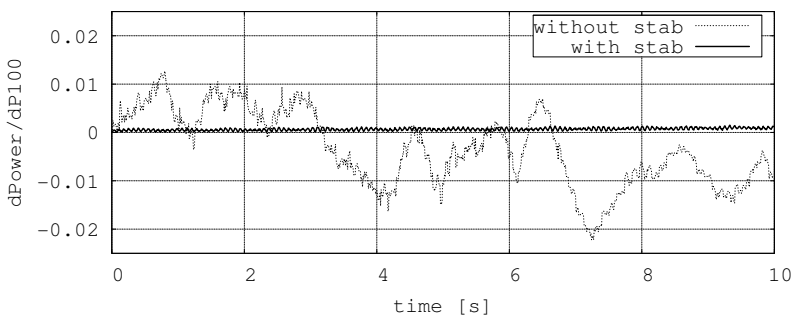

(c) comparison with a big number of time steps

Fig. 20: The coupling power variation using the Hermitian temporal interpolation and the stabilizer force $(\bar{e}=1 \%)$

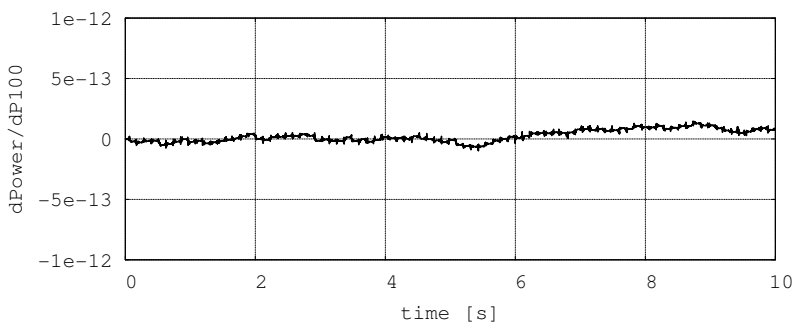

Fig. 21: The coupling power variation using the new algorithm introduced in Section 4 and the stabilizer force $(\bar{e}=1 \%)$

power variation due to the residual force evidenced in Figures 19 is eliminated, as shown by Figure 21.

At the end, the algorithm with Hermitian interpolation of the global velocities was tested with a bigger mesh size and time step ratios. The same analysis introduced in Subsection 3.5 was carried out with the global discretization shown in Figure 4 and a finer local discretization shown in Figure 22 (32 quadrilateral elements through the thickness).
Thus, the following time steps and convergence tolerance were used:

- $\Delta t_{g}=0.4 \cdot 10^{-3} s$ for the global analysis;

$-\Delta t_{l}=0.0125 \cdot 10^{-3} s$ for the local analysis;

$-\bar{e}=1 \%$ as convergence tolerance of the coupling.

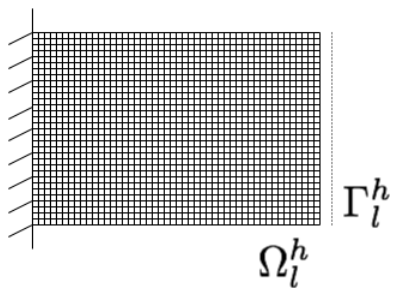

Fig. 22: Discretization of the local problem: the mesh size ratio with the global problem is equal to 32

The results in terms of stability are shown in Figure 23 in comparison with mesh size and time step ratios equal to 4 and in Figure 24 in comparison with the domain decomposition performed with equivalent discretizations.

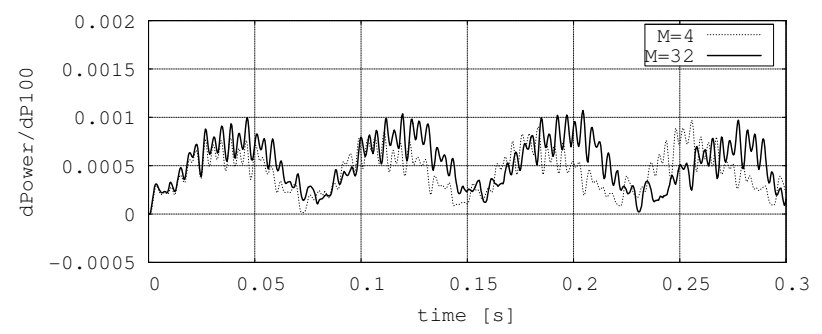

Fig. 23: The residual power variation using the Hermitian temporal interpolation and the stabilizer force with $M=32$ $(\bar{e}=1 \%)$

Although the curves in Figure 23 oscillates around comparable values despite the different mesh size ratio and the methodology, further studied could be conducted in order to understand the nature of the coupling power variation.

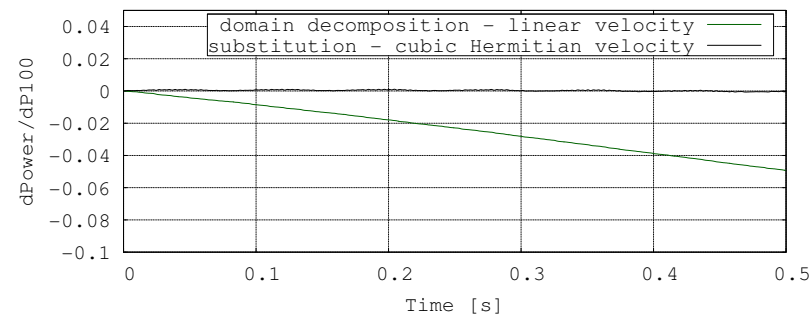

Fig. 24: Comparison between the domain decomposition and substitution with bigger mesh size and time step ratios 


\section{Conclusion}

In this paper, we presented the bases of a substitution approach which enables one to keep the global model and associated time step unchanged.

The next step will be to apply this method to the propagation of large delaminations of the type encountered in laminated structures under impact. A first study was presented in [15], where a domain decomposition method proposed in [10] was successfully tested on a two-dimensional delamination problem. In this case, an internal study by Victor Oancea of Simulia in Providence estimated that the proposed method could lead to at least a tenfold time saving (in addition to simplifications in the database and the coding). In order to do that, the number of iterations must be kept as small as possible. A first option, as proposed in [18], is to try to use a nonintrusive version of a quasi-Newton method [6]. A second option, as proposed in [17], is to use improved mixed boundary conditions for the connection between the global domain and the local domain.

Acknowledgements The authors wish to thank Victor Oancea Simulia in Providence for his fruitful comments and support.

\section{A Connectivity matrices and spatial operators}

With reference to Figure 3 and numbering nodes firstly from the bottom to the top and secondly from left to right, the connectivity matrices for the domain decomposition method write:

$\mathbf{C}_{1}=\left[\begin{array}{llllll}\mathbf{0}_{10} & \mathbf{0}_{10} & \mathbf{0}_{10} & \mathbf{0}_{10} & \mathbf{0}_{10} & \mathbf{I}_{10}\end{array}\right]$

$\mathbf{C}_{2}=\left[\begin{array}{ll}\mathbf{I}_{4} & \mathbf{0}_{4}\end{array}\right]$

where $\mathbf{0}_{2 n}$ is a squared matrix of zeros of dimension $2 n$ and $\mathbf{I}_{2 n}$ is an identity matrix of dimension $2 n$.

With reference to figures 4 and 5 and numbering nodes firstly from the bottom to the top and secondly from left to right, the connectivity matrices write:

$\mathbf{C}_{g}=\left[\begin{array}{lll}\mathbf{0}_{4} & \mathbf{I}_{4} & \mathbf{0}_{4}\end{array}\right]$

$\mathbf{C}_{l}=\left[\begin{array}{llllll}\mathbf{0}_{10} & \mathbf{0}_{10} & \mathbf{0}_{10} & \mathbf{0}_{10} & \mathbf{0}_{10} & \mathbf{I}_{10}\end{array}\right]$

with the same notation mentioned above in the appendix.

Furthermore, the incompatibility between the meshes both in domain decomposition and in substitution leads to the definition of projection and interpolation operators, used to impose constraints or assign values at the nodes not in common. So, referred to position parameters $y_{i}$ and $h$ in Figure 25, the projection operator writes:

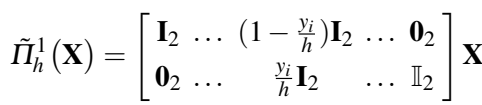

and the interpolation operator simply writes:

$\Pi_{h}^{1}(\mathbf{X})=\left[\begin{array}{ccccc}\mathbf{I}_{2} & \ldots & \left(1-\frac{y_{i}}{h}\right) \mathbf{I}_{2} & \ldots & \mathbf{0}_{2} \\ \mathbf{0}_{2} & \ldots & \frac{y_{i}}{h} \mathbf{I}_{2} & \ldots & \mathbb{I}_{2}\end{array}\right]^{T} \mathbf{X}$

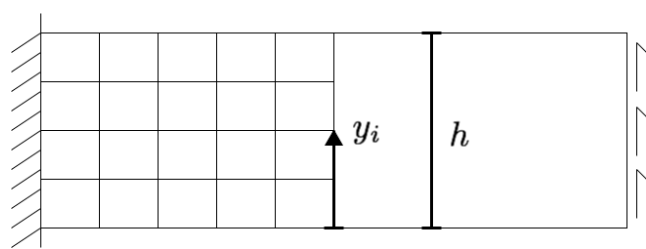

Fig. 25: The position along the interface that determines the values in the projection and interpolation operators

Considering the homogeneous structured mesh, the two Equations (57) and (58) simply become:

$$
\begin{aligned}
& \tilde{\Pi}_{h}^{1}(\mathbf{X})=\left[\begin{array}{lllll}
\mathbf{I}_{2} & \frac{3}{4} \mathbf{I}_{2} & \frac{1}{2} \mathbf{I}_{2} & \frac{1}{4} \mathbf{I}_{2} & \mathbf{0}_{2} \\
\mathbf{0}_{2} & \frac{1}{4} \mathbf{I}_{2} & \frac{1}{2} \mathbf{I}_{2} & \frac{3}{4} \mathbf{I}_{2} & \mathbb{I}_{2}
\end{array}\right] \mathbf{X} \\
& \Pi_{h}^{1}(\mathbf{X})=\left[\begin{array}{lllll}
\mathbf{I}_{2} & \frac{3}{4} \mathbf{I}_{2} & \frac{1}{2} \mathbf{I}_{2} & \frac{1}{4} \mathbf{I}_{2} & \mathbf{0}_{2} \\
\mathbf{0}_{2} & \frac{1}{4} \mathbf{I}_{2} & \frac{1}{2} \mathbf{I}_{2} & \frac{3}{4} \mathbf{I}_{2} & \mathbf{I}_{2}
\end{array}\right]^{T} \mathbf{X}
\end{aligned}
$$

\section{References}

1. O. Allix. A composite damage meso-model for impact problems. Composites Science and Technology, 61:2193-2205, 2001.

2. O. Allix and L. Blanchard. Mesomodeling of delamination: towards industrial applications. Composites Science and Technology, 66(6):731-744, 2006.

3. I. Babuška and T. Strouboulis. The Finite Element Method and its Reliability. Numerical Mathematics and Scientific Computation. Oxford Science Publications: New York, USA, 2001.

4. K.-J. Bathe. Finite Element Procedures. Prentice-Hall, Inc., 1996

5. T. Belytschko and R. Mullen. Stability of explicit-implicit mesh partitions in time integration. International Journal for Numerical Methods in Engineering, 12:1575-1586, 1978.

6. C. G. Broyden. The convergence of a class of double-rank minimization algorithms. Journal of the Institute of Mathematics and Its Applications, 6:76-90, 1970.

7. M. Brun, A. Batti, A. Limam, and A. Gravouil. Explicit/implicit multi-time step co-computations for blast analyses on a reinforced concrete frame structure. Finite Elements in Analysis and Design, 52:41-59, 2012.

8. M. Chevreuil, A. Nouy, and E. Safatly. A multiscale method with patch for the solution of stochastic partial differential equations with localized uncertainties. Computer Methods in Applied Mechanics and Engineering, 255:255-274, 2013.

9. A. Combescure and A. Gravouil. A time-space multi-scale algorithm for transient structural nonlinear problems. Editions scientifiques et medicales, 2(1):43-55, 2001.

10. A. Combescure and A. Gravouil. A numerical scheme to couple subdomains with different time-steps for predominantely transient linear analysis. Computer Methods in Applied Mechanics and Engineering, 191(11-12):1129-1157, 2002.

11. R. Courant, K. Friedrichs, and H. Lewy. On the partial difference equations of mathematical physics. IBM Journal of Research and Development, 11(2):215-234, 1967.

12. P. Cresta, O. Allix, C. Rey, and S. Guinard. Nonlinear localization strategies for domain decomposition methods: Application to post-buckling analyses. Computer Methods in Applied Mechanics and Engineering, 196:1436-1446, 2007.

13. F. Daghia and P. Ladeveze. A micro-meso computational strategy for the prediction of the damage and failure of laminates. Composite Structures, 94(12):3644-3653, 2012. 
14. W. J. T. Daniel. A partial velocity approach to subcycling structural dynamics. Computer Methods in Applied Mechanics and Engineering, 192(3):375-394, 2003.

15. C. Dupleix-Couderc, O. Allix, F. Gatuingt, and B. Malherbe. Towards the multi-scale analysis of delamination in dynamics. ECCM-2010: 4th European Conference on Computational Mechanics: Solids, Structures and Coupled problems, Paris, 2010.

16. D. P. Flanagan and T. Belytschko. Eigenvalues and stable time steps for the uniform strain hexahedron and quadrilateral. Journal of Applied Mechanics, 51(1):35-40, 1985.

17. L. Gendre, O. Allix, and P. Gosselet. A two-scale approximation of the schur complement and its use for non-intrusive coupling. International Journal for Numerical Methods in Engineering, 87:889-905, 2011.

18. L. Gendre, O. Allix, P. Gosselet, and F. Comte. Non-intrusive and exact global/local techniques for structural problems with local plasticity. Computational Mechanics, pages 233-245, 2009.

19. A. Ghanem, M. Torkhani, N. Mahjoubi, T. N. Baranger, and A. Combescure. Arlequin framework for multi-model, multi-time scale and heterogeneous time integrators for structural transient dynamics. Computational Methods in Applied Mechanics and Engineering, 254:292-308, 2013.

20. A. Gravouil and A. Combescure. Multi-time-step explicit-implicit method for non-linear structural dynamics. International Journal for Numerical Methods in Engineering, 50:199-225, 2001.

21. P.-A. Guidault, O. Allix, L. Champaney, and J.-P. Navarro. A two-scale approach with homogeneisation for the computation of cracked structures,. Computer and Structures, 85(17-18):13601371, 2007.

22. P. Gupta, J. P. Pereira, and D. J. Kim et al. Analysis of threedimensional fracture mechanics problems: A non-intrusive approach using a generalized finite element method. Engineering Fracture Mechanics, 90:41-64, 2012.

23. H. D. Hibbitt and B. I. Karlsson. Analysis of a Pipe Whip, EPRI Report, NP-1208, Electric Power Research Institute. The Institute, 1979.

24. T. J. R. Hughes. The Finite Element Method, linear static and dynamic Finite Element Analysis. Prentice-Hall, Inc., 1987.

25. T. J. R. Hughes and W. K. Liu. Implicit-explicit finite element in transient analysis: stability theory. Journal of Applied Mechanics, 45:371-374, 1978.

26. P. Ladeveze, J.-C. Passieux, and D. Neron. The latin multiscale computational method and the proper generalized decomposition. Computer Methods in Applied Mechanics and Engineering, 199(21-22):1287-1296, 2010

27. N. Mahjoubi, A. Gravouil, and A. Combescure. Coupling subdomains with heterogeneous time integertors and incompatible time steps. Computational Mechanics, 44:825-843, 2009.

28. N. Mahjoubi, A. Gravouil, A. Combescure, and N. Greffet. A monolithic energy conservating method to couple heterogeneous time integrators with incompatible time steps in structural dynamics. Computer Methods in Applied Mechanics and Engineering, 200:1069-1086, 2011.

29. N. M. Newmark. A method of computation for structural dynamics. Journal of the Engineering Mechanics Division, 85(7):67-94, 1959.

30. J. Plews, C. A. Duarte, and T. Eason. An improved nonintrusive global-local approach for sharp thermal gradients in a standard fea platform. International Journal for Numerical Methods in Engineering, 91(4):361-397, 2012.

31. A. Prakash and K. D. Hjelmstad. A feti-based multi-time-step coupling method for newmark schemes in structural dynamics. International Journal of Numerical Methods in Engineering, 61:21832204, 2004.

32. E. Pruliere, F. Chinesta, and A. Ammar. On the deterministic solution of multidimensional parametric models using the proper generalized decomposition. Mathematics and Computers in Simulation, 81(4):791-810, 2010.
33. P. Smolinski, T. Belytschko, and M. Neal. Multi-time-step integration using nodal partitioning. International Journal for Numerical Methods in Engineering, 26:349-359, 1988.

34. L. Verlet. Thermodynamical properties of lennard-jones molecules. Computer Experiments on Classical Fluids, 159(1):98-103, 1967.

35. J. D. Whitcomb. Iterative global/local finite element analysis. Computers and Structures, 40(4):1027-1031, 1991.

36. O. C. Zienkiewicz and R. L. Taylor. The Finite Element Method. Butterworth Heinemann, 2000. 